\title{
SMALL DATA SCATTERING FOR A SYSTEM OF NONLINEAR SCHRÖDINGER EQUATIONS
}

\author{
Nakao Hayashi, Chunhua Li and Tohru Ozawa
}

Abstract. We study the scattering theory for a system of nonlinear Schrödinger equations in space dimension $n \geqslant 3$. In the case $n \geqslant 4$, existence of the scattering operator is proved in small data setting in the Sobolev space $H^{n / 2-2}$. In the case $n=3$, a similar result is proved in the weighted $L^{2}$ space $\langle x\rangle^{-1 / 2} L^{2}=\mathscr{F}\left(H^{-1 / 2}\right)$ under the mass resonance condition.

Mathematics subject classification (2010): 35Q55.

Keywords and phrases: nonlinear Schrödinger equations, scattering theory.

\section{REFERENCES}

[1] A. Bachelot, Problème de Cauchy global pour des systèmes de Dirac-Klein-Gordon, Ann. Inst. Henri Poincaré, 48 (1988), 387-422.

[2] J. BERGH AND J. LÖFSTRÖM, Interpolation Spaces, Springer, 1976.

[3] T. Cazenave, Semilinear Schrödinger Equations, Courant Lecture Notes in Mathematics vol. 10, American Mathematical Society (2003).

[4] T. Cazenave And F. B. Weissler, The Cauchy problem for the critical nonlinear Schrödinger equation in $H^{s}$, Nonlinear Analysis TMA, 14 (1990), 807-836.

[5] M. Colin AND T. Colin, On a quasilinear Zakharov system describing laser-plasma interactions, Diff. Integral Equations, 17 (2004), 297-330.

[6] V. Georgiev, Global solution of the system of wave and Klein-Gordon equations, Math. Z., 203 (1990), 683-698.

[7] N. Hayashi, M. IKeda And P. I. NAumkin, Wave operator for the system of the Dirac-KleinGordon equations, to appear in Math. Method. Appl. Sci.

[8] N. HAYASHI, C. Li AND P. I .NAUMKIN, On a system of nonlinear Schrödinger equations in 2d, preprint, Differental Integral Equations, 24 (2011), 417-434.

[9] N. HayAshi, C. Li AND P. I. NAUMKIn, Modified wave operator for a system of nonlinear Schrödinger equations in $2 d$, preprint.

[10] N. HAYASHI AND T. OZAWA, Scattering theory in the weighted $L^{2}\left(\mathbb{R}^{n}\right)$ spaces for some Schrödinger equations, Ann. Inst. H. Poincarè Phys., 48 (1988), 17-37.

[11] T. KATO, On nonlinear Schrödinger equations II, $H^{s}$-solutions and nonconditional well-posedness, J. Anal. Math., 67 (1995), 281-306.

[12] M. KeEl And T. TAO, Endpoint Strichartz estimates, Amer. J. Math., 120 (1998), 955-980

[13] S. Machihara, K. Nakanishi And T. OzaWA, Nonrelativistic limit in the energy space for nonlinear Klein-Gordon equations, Math. Ann., 332 (2002), 603-621.

[14] S. Machihara, K. NAKAnishi And T. OzaWA, Small global solutions and the nonrelativistic limitfor the nonlinear Dirac equation, Rev. Math. Iberoamericana, 19 (2003), 179-194.

[15] M. NAKAMURA AND T. OZAWA, Low energy scattering for nonlinear Schrödinger equations in fractional order Sobolev spaces, Rev. Math. Phys., 9 (1997), 397-410.

[16] K. NAKANISHI, Asymptotically-free solutions for the short-range nonlinear Schrödinger equation, SIAM J. Math. Anal., 32 (2001), 1265-1271.

[17] K. NAKANISHI AND T.OZAWA, Remarks on scattering for nonlinear Schrödinger equations, NoDEA Nonlinear Differential Equations Appl., 9 (2002), 45-68. 
[18] W. StRauss, Nonlinear Wave Equations, CBMS Regional Conference Series in Math., 73, AMS, 1989.

[19] H. TRIEBEL, Theory of Function Spaces, Monographs in Math., 78, Birkhäuser, 1983. 